\title{
Efeito do Processamento da Soja Integral sobre a Energia Metabolizável e a Digestibilidade dos Aminoácidos para Aves ${ }^{1}$
}

\section{Ednardo Rodrigues Freitas ${ }^{2}$, Nilva Kazue Sakomura ${ }^{3}$, Rafael Neme ${ }^{4}$, Andréa Luciana dos Santos ${ }^{4}$, João Batista K. Fernandes ${ }^{5}$}

\begin{abstract}
RESUMO - Essa pesquisa foi desenvolvida para se avaliar o valor nutricional da soja integral submetida a diferentes processamentos. Quatro ensaios de metabolismo foram conduzidos para determinar os coeficientes de digestibilidade da matéria seca (CDMS), do extrato etéreo (CDEE) e dos aminoácidos e os valores de energia metabolizável aparente e verdadeira da soja integral desativada (SID) e extrusada (SIE) e das misturas de farelo de soja com óleo degomado de soja (FSO) ou com óleo ácido de soja (FSOA). Nos ensaios um e dois, utilizou-se a metodologia tradicional de coleta total de excretas com pintos e galos, respectivamente. A metodologia da alimentação forçada com galos adultos intactos foi utilizada no ensaio três, e com galos cecotomizados, no ensaio quatro. Os CDMS e CDEE e a energia metabolizável determinados com galos foram superiores aos determinados com pintos. Os maiores valores de CDMS, CDEE e de energia metabolizável foram obtidos para FSO, seguidos pelos da SIE e FSOA, e os menores, para a SID. O processo de extrusão proporcionou melhores resultados no aproveitamento da gordura do grão de soja e, conseqüentemente, da energia. Entretanto, a digestibilidade dos aminoácidos não foi influenciada pelos processamentos. Os diferentes processamentos conferiram à soja integral características nutricionais que se distinguiram, principalmente quanto ao valor de energia metabolizável, que também variou com a idade das aves.
\end{abstract}

Palavras-chave: extrusão, galos, pintos, processamento térmico, soja desativada

\section{Effects of Processing on Full Fat Soybean Metabolizable Energy Determined by Different Methodologies and Digestibility of Amino Acids for Poultry}

\begin{abstract}
This work was conducted to evaluate the nutritional value of whole soybean submitted to different processing. Four trials were conducted to determine digestibility coefficients of dry matter (DCDM), ether extract (DCEE) and amino acids and the values of apparent and true metabolizable energy of the whole deactivated soybean processed by steam heated (WDS) and whole extruded soybean (WES) compared to a mixture of soybean meal plus soybean oil (SMO) and soybean meal plus soybean acid oil (SMAO). The trials one and two were conducted by using the total collection of excreta method with chicks and roosters, respectively. In the trials three and four, the force-feeding method with intact and cecectomized roosters, respectively, were used. The DCDM, DCEE and metabolizable energy determined by using roosters were higher than that determined with chicks. The DCDM, CDEE and metabolizable were higher for SMO, followed by WSE, SMAO and WDS. The DCMS, DCEE and metabolizable energy of WSE were higher than those of WDS. The amino acids digestibility coefficients did not differ for soybeans. The processing of whole soybeans promoted different values in the metabolizable energy of soybeans, which varied also with the bird age.
\end{abstract}

Key Words: chick, deactivated soybean, extrusion, heat processing, rooster

\section{Introdução}

A soja integral processada, em razão de sua alta qualidade protéica e alta concentração energética, tornou-se uma matéria-prima importante para a alimentação de aves. Entretanto, a necessidade de processamento para inativar os fatores antinutricionais presentes nos grãos crus e a manutenção da qualidade nutricional impulsionaram o desenvolvimento de vários métodos para se atingir esse objetivo, originando produtos com características nutricionais variáveis (Jorge Netto, 1992; Navarro, 1992).

Segundo Waldroup (1982), os processamentos da soja integral mais importantes comercialmente são a tostagem e a extrusão. Jorge Netto (1992) relacionou sete métodos de processamento da soja integral: tostagem por tambor rotativo, tostagem por vapor úmido, tostagem por vapor seco, tostagem por "jet sploder", micronização, extrusão úmida ou seca e microondas. Porém, em função do custo do

\footnotetext{
1 Parte da tese de Doutorado do primeiro autor apresentada à FCAV,UNESP, Jaboticabal.

2 Eng. Agrônomo DZ/CCA/UFC - Campus do Pici, Caixa Postal - 12.168, CEP - 60.355-970, Fortaleza, Ceará. E.mail: ednardo@ufc.br

${ }^{3}$ Profa. do Departamento de Zootecnia da FCAV/UNESP - Jaboticabal, SP. Via de Acesso Professor Paulo Donato Castellane, s/n, CEP: 14884-900, Jaboticabal. Email: sakomura@fcav.unesp.br.

${ }^{4}$ Zootecnista, aluno do curso de Pós-Graduação em Zootecnia da FCAV/UESP/Jaboticabal.

5 Zootecnista - Centro de Aqüicultura da UNESP, Jaboticabal, SP.
} 
processamento do grão de soja, que acaba influenciando o custo final do produto comercial, sempre surge o interesse por outros processos, entre os quais está a desativação do grão da soja ou pré-cozimento, cujo produto comercial é a soja integral desativada. Nesse processamento, os grãos inteiros de soja são submetidos a vapor $\left(63-107^{\circ} \mathrm{C}\right)$ sob pressão $\left(4-8 \mathrm{kgf} / \mathrm{cm}^{2}\right)$ e vácuo, para inativação dos fatores antinutricionais, e os custos por tonelada de produto processado são relativamente menores em relação a outros normalmente utilizados com o mesmo objetivo (Freitas, 2003).

Estudos (Jorge Netto, 1992; Navarro, 1992;Zanella et al., 1999; Café et al., 2000; Sakomura et al. 2004) têm demonstrado que o valor nutricional da soja integral é afetado pelo processamento, justificando a importância da determinação do valor nutricional desse produto para alimentação de aves. De acordo com Sakomura et al. (2004), entre outros fatores, a idade dos frangos de corte influenciou a digestibilidade dos nutrientes e o aproveitamento da energia das sojas integrais processadas, sendo que o aproveitamento da energia dos alimentos aumentou com a idade das aves.

A energia metabolizável dos alimentos pode variar com a linhagem das aves (Yaghobfar, 2001) e o método de alimentação (Askbrant, 1988) utilizados nos ensaios de metabolismo. Segundo Franchesch et al. (2002), deve existir uma preocupação constante com a conveniência de se utilizar um tipo de ensaio de metabolismo específico para avaliar a digestibilidade da energia do alimento para aves.

Além do correto valor energético, as informações sobre a digestibilidade dos aminoácidos dos alimentos podem ser imprescindíveis para que os nutricionistas maximizem o retorno econômico da produção de aves, ao formularem as rações com os valores de aminoácidos digestíveis (Fischer Jr. et al., 1998; Rodrigues et al., 2002).

Nesse contexto, esta pesquisa foi realizada para se determinar o efeito dos processamentos da soja integral sobre os valores de energia metabolizável, determinados por diferentes métodos, e a digestibilidade da matéria seca, do extrato etéreo e dos aminoácidos.

\section{Material e Métodos}

Foram avaliados dois tipos de sojas integrais, produtos comerciais disponíveis para alimentação animal com a denominação de soja integral desativada (SID) e soja integral extrusada (SIE) (CAROL, São
Joaquim da Barra, SP), processadas segundo descrição apresentada por Freitas (2003). O farelo de soja foi reconstituído com óleo de soja (FSO) e óleo ácido de soja (FSOA), para comparação com as sojas integrais. A mistura do farelo de soja e óleo foi feita na proporção de $80 \%$ de farelo com $20 \%$ do respectivo óleo, com base na matéria natural.

Amostras dos alimentos foram analisadas para determinação da composição proximal. Os teores de energia bruta foram determinados em bomba colorimétrica adiabática (Modelo "MS 10 A" da Reichel \& Partner GmbH, Alemanha) e as análises de aminoácidos foram realizadas utilizando-se HPLC (High Performance Liquid Cromatography) e metodologia descrita pela AOAC (2000).

O controle de qualidade da soja integral e do farelo de soja foi realizado pelo teste da atividade ureática e solubilidade da proteína. A atividade da urease foi determinada segundo metodologia recomendada pela ANFAR (1992) e expressa pela diferença de $\mathrm{pH}$ da amostra e do "branco", enquantoa solubilidade da proteína em hidróxido de potássio $(\mathrm{KOH})$ a $0,2 \%$, foi estimada conforme metodologia proposta por Araba \& Dale (1990).

Para determinação dos valores de energia metabolizável e dos coeficientes de digestibilidade, foram conduzidos quatro ensaios de metabolismo com diferentes metodologias. No primeiro e segundo ensaios, utilizou-se o método tradicional de coleta total de excretas, adotando-se períodos experimentais de oito dias (três de adaptação às rações e cinco para coleta das excretas).

No primeiro ensaio, 300 machos Cobb de 12 a 21 dias de idade foram alojados em baterias e distribuídos em um delineamento inteiramente casualizado com cinco tratamentos e seis repetições de 10 aves. No segundo, 70 galos adultos intactos Isa Browm com 85 semanas de idade e peso médio de $3.170 \pm 204 \mathrm{~g}$ foram alojados em gaiolas de arame e distribuídos em delineamento inteiramente casualizado com cinco tratamentos, cada um com sete repetições de duas aves.

Os tratamentos consistiram de dois tipos de soja integral processada, extrusada (SIE) e desativada (SID), de misturas de farelo de soja com óleo de soja degomado (FSO) e óleo ácido de soja (FSOA), e uma ração-referência (Tabela 1).

Os alimentos substituíram, com base na matéria natural, $40 \%$ da ração-referência, de modo que a ração-teste era composta por $60 \%$ da ração-referência e $40 \%$ de um dos alimentos testados. 
Tabela 1 - Composições percentual e calculada da ração-referência

Table 1 - Ingredient and calculated compositions of reference diet

Ingrediente

$(\%)$

Ingredient

\begin{tabular}{|c|c|}
\hline Milho & 63,716 \\
\hline Corn & \\
\hline Farelo de soja & 32,432 \\
\hline $\begin{array}{l}\text { Soybean meal } \\
\text { Calcário }\end{array}$ & 1,027 \\
\hline Limestone & \\
\hline $\begin{array}{l}\text { Fosfato bicálcico } \\
\text { Dicalcium phosphate }\end{array}$ & 1,694 \\
\hline $\begin{array}{l}\text { Mistura mineral } \\
\text { Mineral mix }\end{array}$ & 0,050 \\
\hline $\begin{array}{l}\text { Mistura vitamínica } \\
\text { Vitamin mix }^{2}\end{array}$ & 0,300 \\
\hline $\begin{array}{l}\mathrm{L}-\text { lisina } \mathrm{HCl} \\
\text { L-lysine - } \mathrm{HCl}\end{array}$ & 0,175 \\
\hline $\begin{array}{l}\text { DL-metionina }(99 \%) \\
\text { DL-methionine }\end{array}$ & 0,204 \\
\hline $\begin{array}{l}\text { Sal comum } \\
\text { Salt }\end{array}$ & 0,402 \\
\hline Total & 100,00 \\
\hline
\end{tabular}

Composição calculada

Calculated composition

\begin{tabular}{|c|c|}
\hline Energia metabolizável (kcal/kg) & 2.900 \\
\hline Metabolizable energy & \\
\hline Proteína bruta $(\%)$ & 20,517 \\
\hline Crude protein & \\
\hline Lisina $(\%)$ & 1,198 \\
\hline Lysine & \\
\hline Metionina $(\%)$ & 0,521 \\
\hline Methionine & \\
\hline Metionina + cistina $(\%)$ & 0,850 \\
\hline Methionine + Cystine & \\
\hline Cálcio $(\%)$ & 0,908 \\
\hline Calcium & \\
\hline $\begin{array}{l}\text { Fósforo disponível (\%) } \\
\text { Available phosphorus }\end{array}$ & 0,426 \\
\hline $\begin{array}{l}\text { Sódio }(\%) \\
\text { Sodium }\end{array}$ & 0,201 \\
\hline
\end{tabular}

${ }^{1}$ Composição por kg do produto (Composition per kg of product): $\mathrm{Mn}$ - 150.000 mg; Zn - 100.000 mg; Fe 100.000 mg; Cu - 16.000 mg; I - $1.500 \mathrm{mg}$

${ }^{2}$ Composição por kg do produto (Composition per kg of product): - Vit. A - 2.666.000 UI; Vit. B1 - $600 \mathrm{mg}$; Vit. B2 - $2.000 \mathrm{mg}$; Vit. B6 - 933,10 mg; Vit. B12 - 4.000 mcg; Vit. D3 - 666,50 mg; Vit. E 5.000 UI; Vit. K - 600 mg; Ácido fólico (Folic acid) - 333,25 mg; Ácido pantotênico (Pantothenic acid) - 5.000 mg; Biotina (Biotin) $20 \mathrm{mg}$; Colina (Choline) - $133.330 \mathrm{mg}$; Niacina (Niacine) - $13.333 \mathrm{mg}$; Selênio (Selenium) -100 mg; Antioxidante (Antioxidant) - 7,5 g; Coccidiostático (Coccidiostatic) - 33,332 g; Promotor de crescimento (Growth promoter) - 20 g; Veículo Q.S.P (Inert filler) - $1000 \mathrm{~g}$.
Durante todo o período experimental, água e ração foram fornecidas à vontade, abastecendo-se os comedouros com ração três vezes ao dia, para evitar desperdícios. Para a coleta das excretas, foram instaladas, sob as gaiolas, bandejas revestidas com plástico para evitar perdas.

A identificação das excretas provenientes dos alimentos em avaliação foi realizada com a adição de $1 \%$ de óxido férrico nas rações, no primeiro e no último dia de coleta. Duas vezes ao dia, realizou-se a coleta das excretas, que, após serem acondicionadas em sacos plásticos, foram congeladas. No final do período experimental, após o descongelamento à temperatura ambiente, as excretas de cada repetição foram homogeneizadas, para a retirada de uma amostra, que foi seca em estufa de ventilação forçada a $55^{\circ} \mathrm{C}$, por 72 horas. Em seguida, as amostras foram moídas e encaminhadas ao laboratório, junto com as amostras das rações experimentais, para cálculo dos teores de matéria seca, nitrogênio e extrato etéreo, segundo metodologia descrita por Silva \& Queiroz (2002). Os teores de energia bruta foram determinados em bomba calorimétrica adiabática (Modelo "MS 10 A" da Reichel \& Partner GmbH, Alemanha).

A partir dos resultados laboratoriais, foram calculados os coeficientes de digestibilidade aparente da matéria seca (CDMSA) e de extrato etéreo (CDEEA) e os valores de energia metabolizável aparente (EMA) e aparente corrigida (EMAn), utilizando-se as equações propostas por Matterson et al. (1965).

No terceiro e quarto ensaios, adotou-se a metodologia da alimentação forçada (Sibbald, 1976) com galos adultos Isa Brown, com 87 semanas de idade. No terceiro ensaio, 50 galos intactos com peso de $3.200 \pm 200 \mathrm{~g}$ foram distribuídos em um delineamento experimental inteiramente casualizado, com cinco tratamentos e cinco repetições de duas aves. No quarto ensaio, 30 galos cecotomizados com peso de $2.975 \pm 190 \mathrm{~g}$ foram distribuídos ao acaso em cinco tratamentos, com três repetições de duas aves cada.

Os galos foram submetidos ao experimento 40 dias após a cirurgia, realizada segundo os procedimentos cirúrgicos apresentados por Pupa et al. (1998). 
Nos dois ensaios, os tratamentos consistiram dos alimentos avaliados no primeiro ensaio e de um grupo de aves submetidas a jejum, para determinação das perdas endógenas e metabólicas.

Antes do início do experimento, os galos foram submetidos, durante cinco dias, à adaptação às gaiolas, onde receberam ração e água à vontade. Também no início desse período, sob anestesia local com pomada xilocaína, foi suturada com fio de náilon, junto à pele da cloaca, uma argola plástica com diâmetro de $2,5 \mathrm{~cm}$, que funcionou como adaptador para um saquinho plástico, para a coleta das excretas. A fixação do saco plástico foi feita com o uso de uma borracha tipo atilho, dobrada sobre si, para atingir a pressão necessária.

Antes da ingestão forçada dos alimentos, os galos foram submetidos a jejum de 24 horas, para a limpeza do trato digestivo. Posteriormente, foram forçados a ingerir $30 \mathrm{~g}$ dos alimentos, fornecidos em duas vezes, às 8 e $16 \mathrm{~h}$, para evitar que os galos regurgitassem o alimento fornecido. A alimentação forçada foi realizada com o auxílio de um funil introduzido diretamente no papo das aves. Paralelamente, um grupo permaneceu em jejum.

Depois de alimentados, os galos foram conduzidos às suas respectivas gaiolas, iniciando-se a coleta de excretas imediatamente, com a colocação do saco plástico coletor. Por segurança, foram colocadas bandejas coletoras sob as gaiolas. Ao mesmo tempo, foi iniciada a coleta de excretas do grupo em jejum.

A coleta das excretas foi realizada durante 56 horas e encerrada exatamente 48 horas após a última ingestão forçada de cada alimento. O intervalo de coletas foi de, aproximadamente, 12 horas. Uma vez coletadas, as excretas foram congeladas. No final do período experimental, após descongelamento à temperatura ambiente, foram determinadas as quantidades de excretas produzidas em cada repetição.

No terceiro ensaio, as excretas foram homogeneizadas e submetidas aos mesmos procedimentos de preparação das amostras e mesma metodologia de análises utilizados no primeiro ensaio. Entretanto, no quarto ensaio, a pré-secagem foi realizada por liofilização, durante 48 horas, com placas de aquecimento de $35^{\circ} \mathrm{C}$. Depois de secas, as amostras foram moídas e encaminhadas ao laboratório junto com as amostras dos alimentos, para determinação dos teores de matéria seca e nitrogênio, segundo Silva \& Queiroz (2002), e de aminoácidos, utilizando-se HPLC (High Performance Liquid Cromatography) e metodologia descrita pela AOAC (2000).
Foram calculados os CDMSA, CDEEA e a EMA e EMAn. Também foram calculados os coeficientes de digestibilidade verdadeira da matéria seca (CDMSV) e do extrato etéreo (CDEEV) e os valores de energia metabolizável verdadeira (EMV) e verdadeira corrigida para nitrogênio (EMVn). Também, foram confeccionados os aminogramas dos alimentos e calculados os coeficientes de digestibilidade verdadeiro de cada aminoácido, de acordo com Sibbald (1976).

As análises estatísticas foram realizadas utilizando-se do pacote estatístico SAS (1996). Os dados de CDMSA, CDEEA, EMA e EMAn obtidos nos três primeiros ensaios foram analisados pelo procedimento GLM, segundo um modelo fatorial (quatro alimentos $\mathrm{x}$ três metodologias). Os valores de CDMSV, CDEEV, EMV e EMVn (terceiro ensaio) e os coeficientes de digestibilidade verdadeira dos aminoácidos foram analisados pelo procedimento ANOVA, segundo um modelo inteiramente casualizado. Em todas as análises, as médias foram comparadas pelo teste Tukey (5\%).

\section{Resultados e Discussão}

$\mathrm{Na}$ análise de controle de qualidade do processamento das sojas (Tabela 2), observou-se que os tipos de soja avaliados foram submetidos a processamentos térmicos adequados, estando de acordo com os padrões da ANFAR (1992).

Quanto à composição proximal e de aminoácidos e aos valores de energia bruta dos alimentos (Tabelas 2 e 3), observou-se que os tipos de soja estudados apresentaram pequenas variações em relação aos valores encontrados na literatura (NRC, 1994; Fischer Jr. et al., 1998; Rostagno et al., 2000; Rodrigues et al., 2002). Essas variações podem ser atribuídas ao fato de que a composição dos alimentos de origem vegetal pode ser influenciada por fatores como solo, clima e variedade genética (Albino et al., 1992; Fischer Jr. et al., 1998). Para o uso da soja integral na alimentação de aves, é fundamental considerar o tipo de processamento envolvido (Navarro, 1992).

$\mathrm{Na}$ análise dos resultados obtidos nos ensaios de digestibilidade, observou-se interação significativa entre os alimentos e os métodos pelos quais foram determinados os CDMSA e os CDEEA (Tabela 4) e diferenças significativas entre os CDMSV e os CDEEV dos alimentos pelo método da alimentação forçada (Tabela 5). 
Tabela 2 - Composição química, valores de energia bruta e controle de qualidade das sojas integrais processadas e do farelo de soja

Table 2 - Chemical composition, values of gross energy and quality control of processed soybeans and soybean meal

\begin{tabular}{|c|c|c|c|}
\hline & $\begin{array}{c}\text { Farelo de soja } \\
\text { Soybean meal }\end{array}$ & $\begin{array}{l}\text { Soja integral desativada } \\
\text { Whole deactivated soybean }\end{array}$ & $\begin{array}{l}\text { Soja integral extrusada } \\
\text { Whole extruded soybean }\end{array}$ \\
\hline Matéria seca $(\%)^{1}$ (Dry matter) & 88,33 & 88,45 & 93,25 \\
\hline Extrat etéreo $(\%)^{1}$ (Ether extract) & 1,91 & 20,97 & 19,36 \\
\hline Fibra bruta $(\%)^{1}$ (Crude fiber) & 6,72 & 5,43 & 4,83 \\
\hline Matéria mineral $(\%)^{1}(A s h)$ & 6,85 & 5,30 & 4,83 \\
\hline Fósforo (\%) (Phosphorus) & 0,79 & 0,70 & 0,59 \\
\hline Potássio $(\%)^{1}$ (Potassium) & 2,54 & 1,96 & 1,88 \\
\hline Magnésio (\%) 1 (Magnesium) & 0,36 & 0,28 & 0,29 \\
\hline Sódio $(\%)^{1}$ (Sodium) & 0,024 & 0,031 & 0,024 \\
\hline Atividade da urease ( $\mathrm{D} \mathrm{pH})^{2}$ (Urease activity) & 0,08 & 0,05 & 0,05 \\
\hline
\end{tabular}

${ }^{1}$ Valores expressos com base na matéria seca (values on dry matter basis).

${ }^{2} \mathrm{D} \mathrm{pH}=$ diferença de $\mathrm{pH}$ ( $\mathrm{pH}$ difference).

3 Solubilidade em $\mathrm{KOH}$ (Solubility in $\mathrm{KOH}$ ).

Tabela 3 - Composição de aminoácidos (\%) das sojas integrais processadas e das misturas do farelo de soja com óleo de soja

Table 3 - Amino acid composition (\%) of processed soybeans and mixture of soybean meal and soybean oil

\begin{tabular}{|c|c|c|c|c|}
\hline $\begin{array}{l}\text { Aminoácido } \\
\text { Amino acid }\end{array}$ & $\begin{array}{l}\mathrm{SID}^{2} \\
W D S\end{array}$ & $\begin{array}{l}\mathrm{SIE}^{3} \\
W E S\end{array}$ & $\begin{array}{l}\mathrm{FSO}^{4} \\
S M O\end{array}$ & $\begin{array}{c}\text { FSOA }^{5} \\
\text { SMOA }\end{array}$ \\
\hline Ac. aspártico (Aspartic acid) & 4,50 & 4,28 & 3,32 & 4,61 \\
\hline Ac. glutâmico (Glutamic acid) & 7,34 & 7,03 & 5,43 & 7,62 \\
\hline Alanina (Alanine) & 1,73 & 1,65 & 1,25 & 1,69 \\
\hline Arginina (Arginine) & 3,43 & 3,24 & 2,57 & 3,58 \\
\hline Cistina (Cystine) & 0,61 & 0,57 & 0,35 & 0,46 \\
\hline Histidina (Histidine) & 0,96 & 0,92 & 0,70 & 0,97 \\
\hline Isoleucina (Isoleucine) & 1,63 & 1,71 & 1,18 & 1,70 \\
\hline Leucina (Leucine) & 3,00 & 2,91 & 2,21 & 3,04 \\
\hline Lisina (Lysine) & 2,44 & 2,33 & 1,72 & 2,41 \\
\hline Metionina (Methionine) & 0,68 & 0,65 & 0,30 & 0,34 \\
\hline Serina (Serine) & 2,02 & 1,88 & 1,49 & 2,09 \\
\hline Valina (Valine) & 1,64 & 1,74 & 1,19 & 1,72 \\
\hline
\end{tabular}

1 Valores expressos com base na matéria seca (Values on dry matter basis).

2 SID (WDS) = soja integral desativada (whole deactivated soybean).

${ }^{3} \mathrm{SIE}(\mathrm{ES})=$ soja integral extrusada (whole extruded soybean).

${ }^{4} \mathrm{FSO}(\mathrm{SMO})=$ mistura farelo de soja e óleo de soja (mixture of soybean meal and soybean oil).

${ }^{5}$ FSOA $(\mathrm{SMAO})=$ mistura farelo de soja e óleo ácido de soja (mixture of soybean meal and soybean acid oil).

Os CDMSA determinados pelo método Sibbald foram inferiores aos obtidos pelo método tradicional utilizando-se pintos ou galos. Quando foram feitas as correções para as perdas endógenas de matéria seca, obtendo-se o CDMSV (Tabela 5), o método Sibbald apresentou valores de digestibilidade próximos aos valores de CDMSA estimados pelos outros métodos. O aumento na digestibilidade da matéria seca com as correções para as perdas endógenas pode ser atribuído à subtração da fração endógena de matéria seca do total excretado, diminuindo proporcionalmente a excreção originária do alimento e aumentando o coeficiente de digestibilidade (Albino et al., 1992). 
Tabela 4 - Coeficientes de digestibilidade aparente da matéria seca (CDMSA) e do extrato etéreo (CDEEA) dos alimentos, determinados por diferentes metodologias*

Table 4 - Aparrent digestibility coefficients of dry matter (ADCDM) and ether extract (ADCEE) of feedstuffs determined by different methodologies*

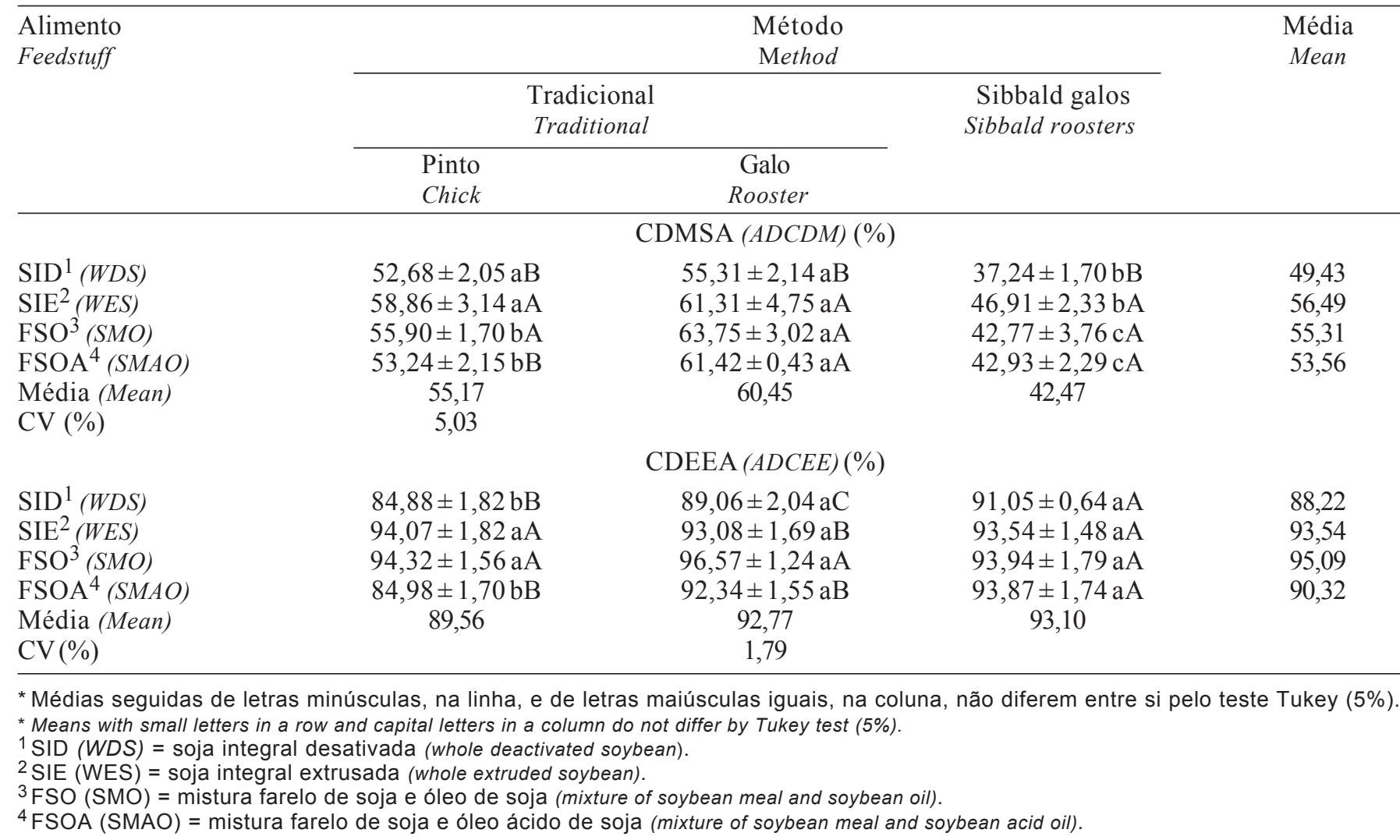

No método tradicional, as aves adultas apresentaram CDMSA dos alimentos superior aos obtidos com pintos, apenas para FSO e FSOA. A maior digestibilidade da matéria seca obtida com as aves adultas se deve à maior capacidade digestiva dessas aves em relação às aves jovens. Resultados semelhantes foram observados por Café et al. (2000), ao avaliarem diferentes tipos de soja integral processada.

Os CDMSA da SID foram inferiores aos obtidos para SIE e FSO em todas as metodologias (Tabela 4). Essa diferença também foi observada para o CDMSV (Tabela 5). Para a SIE e FSO, os CDMSA determinados pelo método tradicional com galos e pintos e os CDMSV determinados pelo método Sibbald não diferiram significativamente entre si e foram semelhantes aos obtidos por Café et al. (2000), com as mesmas metodologias.

Para a digestibilidade do extrato etéreo, observou-se que os dados apresentaram comportamento semelhante aos da digestibilidade da matéria seca, havendo variações nos CDEEA dos alimentos entre as metodologias e em cada uma delas (Tabela 4). Foram constatadas também diferenças significativas entre os CDEEV dos alimentos pelo método Sibbald (Tabela 5).

As variações nos resultados de digestibilidade do extrato etéreo dos alimentos obtidos com as diferentes metodologias, além dos efeitos inerentes a cada metodologia, estão relacionadas à disponibilidade da gordura dos alimentos, à composição em ácidos graxos da gordura e à idade das aves utilizadas. Esses fatores, às vezes, agem sozinhos e/ou interagem, tornando os resultados variáveis.

Nas três metodologias, observou-se menor digestibilidade do extrato etéreo da SID em relação ao da SIE e FSO (Tabela 4), o que pode ser atribuído à menor acessibilidade das enzimas à gordura da SID. Segundo Sakomura et al. (1998), o óleo adicionado às misturas está livre e, na SIE, o óleo é exposto pelo processo de extrusão, que promove a ruptura da estrutura celular, liberando a gordura intracelular. A maior acessibilidade da gordura nesses alimentos facilita a digestão e a absorção da mesma. Por outro 
Tabela 5 - Coeficientes de digestibilidade verdadeira da matéria seca (CDMSV) e do extrato etéreo (CDEEV) dos alimentos*

Table 5 - True digestibility coefficients of dry matter (TDCDM) and ether extract (TDCEE) of feedstuffs*

\begin{tabular}{lcc}
\hline Alimento & CDMSV & CDEEV \\
Feedstuff & TDCDM & TDCEE \\
\hline SID $^{1}($ WDS $)$ & $60,60 \pm 1,70 \mathrm{~b}$ & $92,14 \pm 0,64 \mathrm{~b}$ \\
SIE $^{2}($ WES $)$ & $69,02 \pm 2,33 \mathrm{a}$ & $94,52 \pm 1,37 \mathrm{ab}$ \\
FSO $^{3}($ SMO $)$ & $65,08 \pm 3,76 \mathrm{ab}$ & $94,83 \pm 1,52 \mathrm{a}$ \\
FSOA $^{4}($ SMAO) & $65,38 \pm 2,30 \mathrm{a}$ & $95,00 \pm 1,74 \mathrm{a}$ \\
Média & 65,02 & 94,13 \\
CV $(\%)$ & 4,05 & 1,46 \\
\hline
\end{tabular}

* Médias seguidas de letras minúsculas iguais, na coluna, não diferem entre si, pelo teste Tukey (5\%).

* Means with small letters in a column do not differ by Tukey test (5\%).

1 SID (WDS) = soja integral desativada (whole deactivated soybean).

${ }^{2}$ SIE (WES) = soja integral extrusada (whole extruded soybean).

${ }^{3} \mathrm{FSO}(\mathrm{SMO})=$ mistura farelo de soja e óleo de soja (mixture of soybean meal and soybean oil).

${ }^{4}$ FSOA $(\mathrm{SMAO})=$ mistura farelo de soja e óleo ácido de soja (mixture of soybean meal and soybean acid oil).

lado, no processo de desativação da soja com o uso de vapor, não ocorre liberação do óleo armazenado nos lipossomos, onde as enzimas digestivas das aves não são capazes de atuar (Pinheiro, 1993). A menor acessibilidade da gordura também justifica o menor CDEEA obtido para a SIE em relação ao FSO determinado com galos.

No método tradicional com pintos, o menor CDEEA do FSOA, em relação ao da SIE e do FSO, pode ser atribuído à composição em ácidos graxos do óleo ácido de soja, que é composto, basicamente, por ácidos graxos livres. $\mathrm{O}$ aumento da quantidade de ácidos graxos livres em uma fonte de gordura reduz a digestibilidade e a absorção dessa gordura (Wiseman \& Salvador, 1991; Vilá \& Esteve-Garcia, 1996). Segundo Sklan (1979), a absorção de gordura pelas aves é precedida do rompimento (digestão) dos triglicerídeos e favorecida pela presença de monoglicerídeos resultantes desse rompimento, que ativam a secreção de bile, necessária para a formação das micelas, e a presença de ácidos graxos livres dificulta esses processos.

A maior digestibilidade da gordura da SID apresentada pelos galos em relação aos pintos (Tabela 4) pode ser atribuída à menor capacidade digestiva das aves jovens. Segundo Freeman (1984), as aves jovens apresentam menor capacidade de produção de lipase pancreática e deficiência na produção de bile e, por isso, a digestão e a absorção das gorduras são meno- res. Esse quadro é revertido com o avançar da idade (Noy \& Sklan, 1995).

Para o FSOA, apesar de o óleo estar disponível para a digestão, as aves jovens apresentaram menor CDEEA (Tabela 4). Além do efeito da idade, a composição em ácidos graxos do óleo ácido de soja, também pode ter contribuído para o menor aproveitamento da gordura pelos pintos. A ação prejudicial dos ácidos graxos livres presentes no óleo ácido de soja sobre a digestibilidade da gordura foi menor nas aves adultas, que apresentaram maior capacidade de digestão das gorduras.

A análise estatística dos dados indicou interação significativa entre os métodos de determinação da energia metabolizável e os alimentos (Tabela 6). Exceto para a SIE, os valores de EMA determinados pelo método Sibbald foram menores que os obtidos com galos e pintos pelo método tradicional. Essa diferença no valor de EMA é inerente à própria metodologia da alimentação forçada, pois o consumo de alimento é relativamente baixo, gerando valores de EMA subestimados em função da participação proporcionalmente elevada das perdas endógena e metabólica na energia excretada. Pelo método tradicional, com exceção da EMA da SID, os valores de EMA obtidos com pintos foram menores que os obtidos com galos.

Pelo método tradicional, o valor de EMA da SID foi inferior ao da SIE e FSO, tanto com pintos quanto com galos. Observou-se também que a EMA do FSO foi superior à da SIE, de modo que apenas entre os valores obtidos com galos a diferença foi significativa. Esses resultados podem ser atribuídos à maior disponibilidade da gordura nesses alimentos. No caso do FSO, deve-se considerar também a maior proporção de extrato etéreo, uma vez que, na mistura com o farelo de soja, os tipos de óleos foram adicionados na proporção de $20 \%$ da mistura e essa quantidade de óleo foi maior que a proporção de extrato etéreo determinada para os tipos de soja integral avaliados nesse experimento. No método Sibbald, o maior valor de EMA foi observado para a SIE, seguido do obtido para o FSO, FSOA e SID.

Para os valores de EMAn (Tabela 6), foram observadas algumas variações no comportamento dos dados em relação aos obtidos para a EMA. Esses efeitos podem ser atribuídos às diferentes contribuições do balanço de nitrogênio no cálculo da EMAn dos alimentos em cada método. 
Tabela 6 - Valores de energia metabolizável aparente (EMA) e aparente corrigida (EMAn) dos alimentos determinados por diferentes metodologias*

Table 6 - Values of apparent (AME) and corrected apparent (AMEn) metabolizable energy of feedstuffs determined by different methodologies*

\begin{tabular}{lccc}
\hline Alimento & & Método & Method \\
Feedstuff & & & Média \\
\cline { 2 - 4 } & \multicolumn{2}{c}{$\begin{array}{c}\text { Tradicional } \\
\text { Traditional }\end{array}$} & $\begin{array}{c}\text { Sibbald galos } \\
\text { Sibbald roosters }\end{array}$ \\
\cline { 2 - 4 } & Pinto & Galo \\
Chick & Rooster & \\
\hline
\end{tabular}

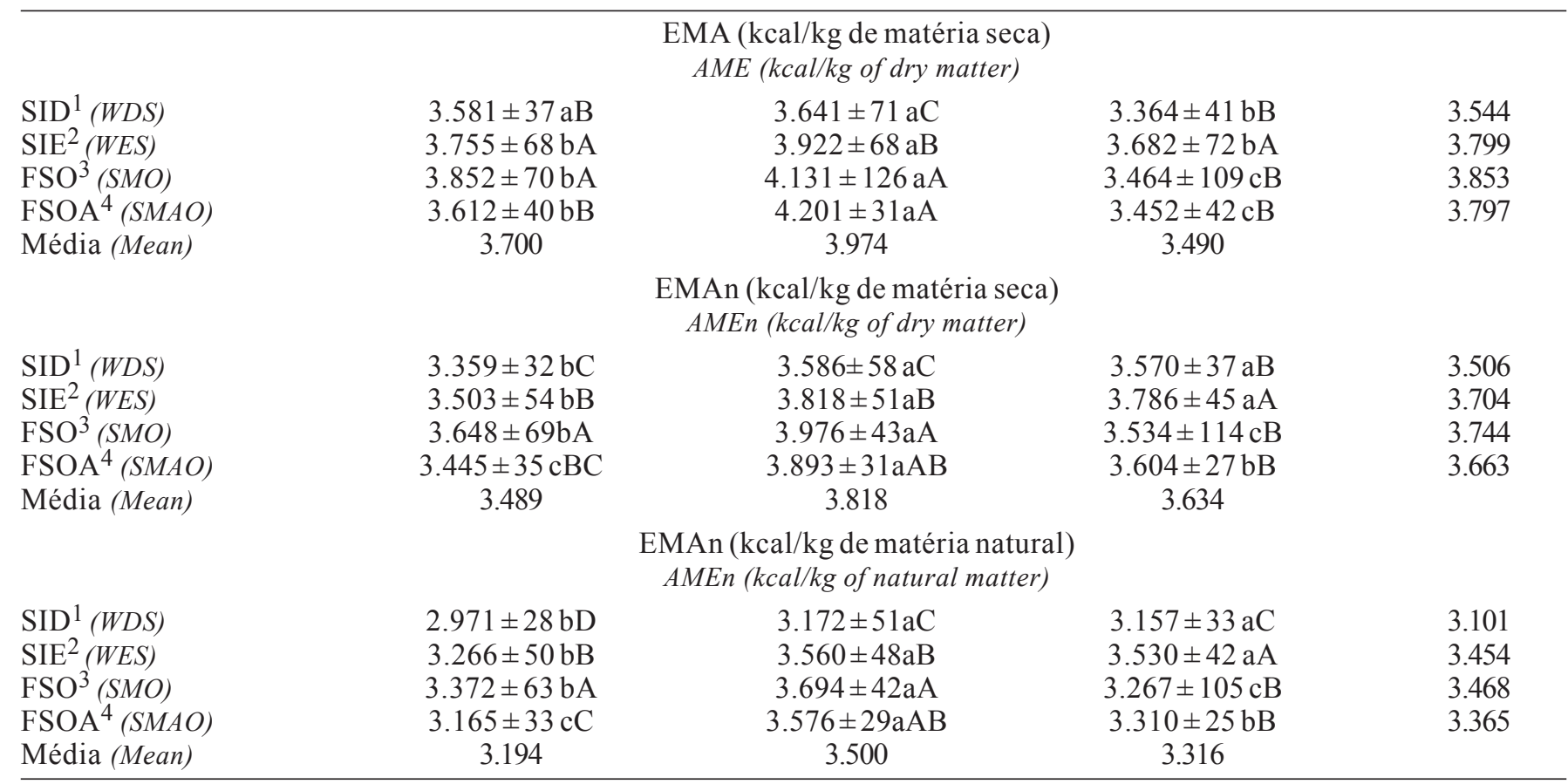

* Médias seguidas de letras minúsculas, na linha, e de letras maiúsculas iguais, na coluna, não diferem entre si pelo teste Tukey (5\%).

* Means with small letters in a row and capital letters in a column do not differ by Tukey test (5\%).

1 SID $(W D S)=$ soja integral desativada (whole deactivated soybean).

2 SIE (WES) = soja integral extrusada (whole extruded soybean).

${ }^{3} \mathrm{FSO}(\mathrm{SMO})=$ mistura farelo de soja e óleo de soja (mixture of soybean meal and soybean oil).

${ }^{4}$ FSOA $(\mathrm{SMAO})=$ mistura farelo de soja e óleo ácido de soja (mixture of soybean meal and soybean acid oil).

A literatura (Albino et al., 1992; Zanella et al., 1999; Café et al., 2000) tem mostrado que as aves jovens utilizadas nos ensaios, por estarem em crescimento, apresentam balanço de nitrogênio positivo e, no cálculo da EMAn, a energia do alimento retida como nitrogênio deve ser descontada da EMA. Isso faz com que os valores de EMAn determinados com pintos sejam menores que os de EMA. Entretanto, nesse experimento, diferente do que tem sido observado, em aves adultas, por outros pesquisadores (Zanella et al., 1999; Café et al., 2000), os galos utilizados no método tradicional apresentaram balanço de nitrogênio positivo, o que também resultou em menores valores de EMAn em relação à EMA. No método Sibbald, pela própria característica do método em utilizar pequena quantidade do alimento, os galos apresentaram balanço de nitrogênio negativo, so- mando-se à EMA a energia do alimento perdida como nitrogênio e aumentando os valores de EMAn em relação à EMA. Por outro lado, segundo Ishibashi \& Yonemochi (2002), a excreção de nitrogênio aumenta com a idade das aves e o nível de proteína do alimento e diminui com o nível de restrição alimentar. Esses fatos justificam a variação na retenção de nitrogênio observada entre o tipo de ave, os alimentos avaliados e a metodologia de alimentação e, conseqüentemente, a diferença entre o valor de EMA determinado com galos em relação ao determinado com pintos, no método tradicional, para a SID, que era apenas numérica, passou a ser significativa, quando se comparou à EMAn. Para as misturas, FSO e FSOA, as diferenças entre as EMAn determinadas com pintos e galos foram menores que entre os valores de EMA. 
Os valores de EMAn dos alimentos, expressos com base na matéria natural, (Tabela 6) apresentaram o mesmo comportamento de quando analisados na matéria seca, porém com variações que se devem às diferenças na quantidade de matéria seca dos alimentos.

Para a EMV e EMVn determinadas pelo método Sibbald (Tabela 7), a análise estatística mostrou diferenças significativas entre os valores de energia dos alimentos.

A SIE apresentou valores de EMV e EMVn significativamente superiores aos obtidos para os demais alimentos. Os valores de EMVn da SIE e FSO foram ligeiramente superiores aos determinados por Café et al. (2000), que utilizaram essa metologia.

Pelo método Sibbald, os valores de EMV foram superiores aos de EMA, o que era esperado, em função das correções resultantes das perdas energéticas e metabólicas endógenas consideradas no cálculo da EMV, resultando em valores superiores. Entretanto, quando se aplicaram as correções para o balanço de nitrogênio, a diferença entre a EMVn e EMAn foi bem menor que entre EMV e EMA. Por outro lado, os valores de EMV foram superiores aos de EMVn, em razão da maior perda de nitrogênio endógeno pelas aves em jejum, em comparação às alimentadas (Dale \& Fuler 1984; Wolynetz \& Sibbald, 1984; Franchesch et al., 2002).

Considerando-se que a digestibilidade da gordura é o fator que mais influencia os valores de energia metabolizável dos alimentos (Carew et al., 1961), certamente os resultados obtidos para a energia metabolizável dos alimentos, pelos diferentes métodos, estão diretamente relacionados aos resultados de CDEEA e CDEEV. Portanto, a disponibilidade da gordura nos alimentos, a composição em ácidos graxos da gordura e a idade das aves são fatores que influenciaram a digestibilidade do extrato etéreo e os valores de energia metabolizável dos alimentos avaliados. Segundo Café et al. (2000), os maiores valores de energia metabolizável obtidos para as sojas integrais submetidas a diferentes processamentos estavam diretamente correlacionados aos maiores valores de digestibilidade do extrato etéreo.

As variações entre os valores de EMA, EMAn e EMVn determinados para as sojas integrais, pelas diferentes metodologias, em relação aos encontrados na literatura, além das diferentes condições experimentais, podem ser atribuídas às condições de processamento, uma vez que, para o mesmo tipo de processamento, não existe completa padronização das condições de temperatura, umidade, tempo e pressão. Moreira et al. (1994) observaram essas variações em três lotes de soja extrusada de mesma procedência.

Os resultados obtidos com diferentes formas de determinação da energia metabolizável indicam que o processamento térmico de pré-cozimento ou desativação da soja integral para originar o produto soja integral desativada (SID) é menos eficiente em disponibilizar a energia do grão para as aves que o processo de extrusão. Por outro lado, deve-se considerar as diferenças dos valores energéticos dos alimentos para as aves em crescimento e adultas, uma vez que, na formulação de rações para aves, há um aumento na

Tabela 7 - Valores de energia metabolizável verdadeira (EMV) e verdadeira corrigida (EMVn) dos alimentos determinados pelo método Sibbald*

Table 7 - Values of true (TME) and corrected true metabolizable energy (TMEn) of feedstuffs determined by Sibbald method*

\begin{tabular}{|c|c|c|c|}
\hline $\begin{array}{l}\text { Alimento } \\
\text { Feedstuff }\end{array}$ & $\begin{array}{c}\text { EMV (kcal/ } \mathrm{kg} \text { de MS }) \\
\text { TME (kcal/kg of dry matter) }\end{array}$ & $\begin{array}{c}\text { EMVn (kcal/kg de MS) } \\
\text { TMEn (kcal/kg of dry matter) }\end{array}$ & $\begin{array}{c}\text { EMVn (kcal/kg de MN) } \\
\text { TMEn (kcal/kg as feed basis) }\end{array}$ \\
\hline$\overline{\operatorname{SID}^{1}(W D S)}$ & $4.074 \pm 41 \mathrm{~b}$ & $3.864 \pm 37 b$ & $3.418 \pm 32 \mathrm{c}$ \\
\hline $\operatorname{SIE}^{2}(W E S)$ & $4.355 \pm 72 \mathrm{a}$ & $4.065 \pm 45 \mathrm{a}$ & $3.790 \pm 42 \mathrm{a}$ \\
\hline $\mathrm{FSOA}^{4}(S M A O)$ & $4.136 \pm 43 b$ & $3.887 \pm 27 b$ & $3.570 \pm 25 b$ \\
\hline Média (Mean) & $4.177 \pm 60$ & $3.908 \pm 70$ & $3.577 \pm 70$ \\
\hline
\end{tabular}

\footnotetext{
* Médias seguidas de letras minúsculas iguais, na coluna, não diferem entre si, pelo teste Tukey (5\%).

* Means with small letters in a column do not differ by Tukey test (5\%).

${ }^{1}$ SID $(W D S)=$ soja integral desativada (whole deactivated soybean).

2 SIE (WES) = soja integral extrusada (whole extruded soybean).

${ }^{3} \mathrm{FSO}(\mathrm{SMO})=$ mistura farelo de soja e óleo de soja (mixture of soybean meal and soybean oil).

${ }^{4}$ FSOA $(\mathrm{SMAO})=$ mistura farelo de soja e óleo ácido de soja (mixture of soybean meal and soybean acid oil).
} 
energia metabolizável dos alimentos para as aves adultas, resultando em menor necessidade de inclusão para atingir o nível energético pretendido.

Neste experimento, não houve diferenças significativas nos coeficientes de digestibilidade verdadeira dos aminoácidos entre os tipos de soja integral avaliados e as misturas do farelo de soja e óleo (Tabela 8). Os resultados obtidos podem ser justificados pelos índices de controle de qualidade dos tipos de soja. A atividade da urease e a solubilidade da proteína de todas as sojas avaliadas encontravam-se dentro do padrão recomendado, indicando que as sojas foram bem processadas. Ao contrário do observado por alguns pesquisadores (Jackson \& Dalibard, 1995; Café et al., 2000), o processo de extrusão não foi mais eficiente em disponibilizar os aminoácidos do grão de soja em relação à desativação por vapor sob vácuo.

Assim como para a energia metabolizável, na literatura, há relatos de variação nos resultados de digestibilidade dos aminoácidos da soja integral sub- metida ao mesmo tipo de processamento, o que pode ser atribuído às diferentes condições experimentais e de processamento. Entretanto, quando se compara a digestibilidade média de todos os aminoácidos, pode-se considerar pequeno o intervalo de variação. Nesse experimento, para a SIE e FSO, alguns aminoácidos apresentaram coeficientes de digestibilidade verdadeiros menores (lisina, cistina, treonina) e outros maiores (metionina, tirosina) que os obtidos por Café et al. (2000). Entretanto, os valores médios de digestibilidade de todos os aminoácidos da SID e SIE foram próximos dos apresentados na literatura (Fischer Jr. et al., 1998; Rostagno et al., 2000; Rodrigues et al., 2002).

A partir dos coeficientes de digestibilidade determinados para cada aminoácido, calculou-se o conteúdo de aminoácidos digestíveis para cada alimento (Tabela 8). Assim como ocorreu com o conteúdo de aminoácidos totais dos alimentos, observou-se variabilidade entre o conteúdo de aminoácidos digestíveis

Tabela 8 - Coeficientes de digestibilidade verdadeira dos aminoácidos (CDV) e conteúdo de aminoácidos digestíveis (AAD) das sojas integrais processadas e das misturas do farelo de soja com óleo

Table 8 - True digestibility coefficients (TDC) and digestibility amino acids content of whole soybean and mixture of soybean meal and soybean oil

\begin{tabular}{|c|c|c|c|c|c|c|c|c|c|}
\hline \multirow[t]{3}{*}{$\begin{array}{l}\text { Aminoácido } \\
\text { Amino acid }\end{array}$} & \multicolumn{2}{|c|}{$\begin{array}{l}\text { SID }^{1} \\
W D S\end{array}$} & \multicolumn{2}{|c|}{$\begin{array}{l}\mathrm{SIE}^{2} \\
W E S\end{array}$} & \multicolumn{2}{|c|}{$\begin{array}{l}\mathrm{FSO}^{3} \\
S M O\end{array}$} & \multicolumn{2}{|c|}{$\begin{array}{l}\text { FSOA }^{4} \\
S M O A\end{array}$} & \multirow[t]{3}{*}{$\mathrm{CV}^{8}(\%)$} \\
\hline & $\mathrm{CDV}^{5}$ & $\mathrm{AAD}^{6,7}$ & $\mathrm{CDV}^{5}$ & $\mathrm{AAD}^{6,7}$ & $\mathrm{CDV}^{5}$ & $\mathrm{AAD}^{6,7}$ & $\mathrm{CDV}^{5}$ & $\mathrm{AAD}^{6,7}$ & \\
\hline & \multicolumn{2}{|c|}{$(\%)$} & \multicolumn{2}{|c|}{$(\%)$} & \multicolumn{2}{|c|}{$(\%)$} & \multicolumn{2}{|c|}{$(\%)$} & \\
\hline Ac. aspártico (Aspartic acid) & 86,80 & 3,91 & 87,79 & 3,76 & 86,66 & 2,88 & 89,96 & 4,15 & 2,32 \\
\hline Ac. glutâmico (Glutamic acid) & 90,24 & 6,62 & 92,21 & 6,49 & 91,01 & 4,94 & 93,85 & 7,15 & 1,97 \\
\hline Alanina (Alanine) & 83,09 & 1,44 & 84,53 & 1,40 & 81,36 & 1,01 & 85,09 & 1,44 & 4,56 \\
\hline Arginina (Arginine) & 90,89 & 3,11 & 91,42 & 2,96 & 90,67 & 2,33 & 93,93 & 3,36 & 2,07 \\
\hline Cistina (Cystine) & 85,43 & 0,52 & 73,49 & 0,42 & 73,10 & 0,26 & 80,09 & 0,37 & 11,66 \\
\hline Fenilalanina (Phenylalanine) & 84,92 & 1,75 & 83,75 & 1,64 & 80,11 & 1,23 & 87,79 & 1,95 & 3,55 \\
\hline Glicina (Glycine) & 81,82 & 1,35 & 80,20 & 1,25 & 75,48 & 0,92 & 78,35 & 1,29 & 4,97 \\
\hline Histidina (Histidine) & 90,13 & 0,87 & 88,37 & 0,82 & 88,48 & 0,62 & 90,66 & 0,88 & 2,40 \\
\hline Isoleucina (Isoleucine) & 85,91 & 1,40 & 89,29 & 1,52 & 87,29 & 1,03 & 89,39 & 1,52 & 2,78 \\
\hline Leucina (Leucine) & 87,64 & 2,63 & 89,22 & 2,59 & 88,38 & 1,95 & 90,46 & 2,75 & 2,35 \\
\hline Lisina (Lysine) & 88,29 & 2,16 & 88,35 & 2,06 & 86,17 & 1,48 & 89,67 & 2,16 & 2,83 \\
\hline Metionina (Methionine) & 94,74 & 0,64 & 94,69 & 0,62 & 90,64 & 0,27 & 95,41 & 0,32 & 3,58 \\
\hline Serina (Serine) & 88,55 & 1,79 & 84,93 & 1,59 & 85,53 & 1,28 & 89,75 & 1,88 & 4,37 \\
\hline Tirosina (Tyrosine) & 90,96 & 1,05 & 92,13 & 1,01 & 93,18 & 0,84 & 93,04 & 1,07 & 2,69 \\
\hline Treonina (Threonine) & 85,18 & 1,29 & 78,66 & 1,14 & 78,45 & 0,87 & 83,92 & 1,32 & 7,45 \\
\hline Triptofano (Tryptophan) & 93,41 & 0,39 & 88,45 & 0,32 & 92,77 & 0,43 & 94,34 & 0,43 & 2,75 \\
\hline Valina (Valine) & 83,19 & 1,36 & 83,20 & 1,45 & 79,71 & 0,95 & 84,43 & 1,46 & 4,41 \\
\hline Média (Mean) & 87,72 & - & 86,51 & - & 85,23 & - & 88,83 & - & \\
\hline
\end{tabular}

\footnotetext{
1 SID $(W D S)=$ soja integral desativada (whole deactivated soybean).

2 SIE (WES) = soja integral extrusada (whole extruded soybean).

${ }^{3} \mathrm{FSO}(\mathrm{SMO})=$ mistura farelo de soja e óleo de soja (mixture of soybean meal and soybean oil).

${ }^{4} \mathrm{FSOA}(\mathrm{SMAO})=$ mistura farelo de soja e óleo ácido de soja (mixture of soybean meal and soybean acid oil).

${ }^{5} \mathrm{CDV}=$ coeficiente de digestibilidade verdadeira (true digestibility coefficients).

${ }^{6} \mathrm{AAD}=$ aminoácidos digestíveis (digestible amino acid).

7 Valores expressos com base na matéria seca (values on dry matter basis).

${ }^{8} \mathrm{CV}=$ coeficiente de variação dos CDV de cada aminoácido (coefficient of variation of each TDC amino acid).
} 
determinados para SIE e FSO e aqueles determinados por Fisher Jr. et al. (1998) e Café et al. (2000). Os resultados obtidos para a SIE também foram variáveis em relação aos obtidos por Rodrigues et al. (2002). Segundo esse autor, essas diferenças estão associadas às variações na quantidade total de cada aminoácido e no coeficiente de digestibilidade, que podem resultar em variações no conteúdo de aminoácidos digestíveis de um mesmo alimento.

\section{Conclusões}

Os diferentes tipos de processamentos conferiram à soja integral características nutricionais que se distinguiram principalmente no teor de energia metabolizável.

$\mathrm{Na}$ formulação de rações para aves utilizando-se soja integral processada, devem-se considerar as diferenças na energia metabolizável, em função do processamento a que foi submetida, e a idade das aves.

O processamento por pré-cozimento é equivalente ao processamento por extrusão em disponibilizar os aminoácidos da soja integral para as aves.

\section{Literatura Citada}

ALBINO, L.F.T.; ROSTAGNO, H.S..; TAFURI, M.L. et al. Determinação dos valores de energia metabolizável aparente e verdadeira de alguns alimentos para aves, usando diferentes métodos. Revista da Sociedade Brasileira de Zootecnia, v.21, n.6, p.1047-1058, 1992

ARABA, M.; DALE, N.M. Evaluation of protein solubility as an indicator of overprocessing soybean meal. Poultry Science, v.69, p.76-83, 1990.

ASKBRANT, S. Metabolizable energy content of rapeseed meal, soybean meal and white-flowered peas determined with laying hens and adult cockerels. British Poultry Science, v.29, p.445-455, 1988.

ASSOCIAÇÃO NACIONAL DOS FABRICANTES DE RAÇÕES. Métodos analíticos de controle de alimentos para uso animal. São Paulo: ANFAR, 1992. p.1-2.

ASSOCIATION OF OFFICIAL ANALYTICAL CHEMISTS AOAC. Official methods of analysis. 17.ed. Washington, D.C.: AOAC, 2000. 1410p.

CAFÉ, M.B.; SAKOMURA, N.K.; JUNQUEIRA, O.M. et al. Determinação do valor nutricional das sojas integrais processadas para aves. Revista Brasileira de Ciência Avícola, v.2, n.1, p.67-74, 2000.

CAREW JR., L.B.; HILL, F.W.; NESHEIN, M.L et al. The value of heated ground unextracted soybean and heated dehulled soybean oil and energy for chick. Journal American Oil Chemistry Society, v.38, p.249-253, 1961.

DALE, N.; FULLER, H.L. Correlation of protein content of feedstuffs with the magnitude of nitrogen correction in true metabolizable energy determination. Poultry Science, v.6, p.1008-1012, 1984
FISCHER JR., A.A.; ALBINO, L.F.T.; ROSTAGNO, H.S. et al. Determinação dos valores de energia metabolizável de alguns alimentos usados na alimentação de aves. Revista Brasileira de Zootecnia, v.27, n.2, p.314-318, 1998.

FRANCHESCH, M.; BERNARD, K.; McNAB, J.M. Comparison of two direct bioassays using 3-week-old broilers to measure the metabolizable energy of diets containing cereals high in fiber: differences between true and apparent metabolizable energy values. British Poultry Science, v.44, p.580-587, 2002.

FREEMAN, C.P. The digestion, absorption and transport of fats in non-ruminants. In: WISEMAN, J. (Ed.). Fats in animal nutrition. London: Butterworths, 1984. p.105-122.

FREITAS, E.R. Avaliação nutricional de alguns alimentos processados para aves por diferentes metodologias e suas aplicações na formulação de rações para frangos de corte. Jaboticabal: Universidade Estadual Paulista, 2003. 129p. Tese (Doutorado em Zootecnia) - Universidade Estadual Paulista, 2003.

ISHIBASHI, T.; YONEMOCHI, C. Possibility of amino acid nutrition in broiler. Animal Science Journal, v.73, p.155-165, 2002.

JACKSON, D.A.; DALIBARD, P. The measurement and validation of the technique of using digestible amino acids for diet formulation in poultry and swine. In: SIMPÓSIO LATINO AMERICANO DE NUTRIÇÃO DE SUÍNOS E AVES, 1995, Campinas. Anais... Campinas: Colégio Brasileiro de Nutrição Animal, 1995. p.47-72.

JORGE NETTO, G. Soja integral na alimentação de aves e suínos. Avicultura Industrial, v.82, n.988, p.4-15, 1992.

MATTERSON, L.D.; POTTER, L.M.; STUTZ, N.W. et al. The metabolizable energy of feed ingredients for chickens. Storrs: The University of Connecticut, Agricultural Experiment Station, 1965. 11p. (Research Report, 7).

MOREIRA, I.; ROSTAGNO, H.S.; COELHO, D.T. et al. Determinação dos coeficientes de digestibilidade, valores energéticos e índices de controle de qualidade do milho e soja integral processados a calor. Revista Brasileira de Zootecnia, v.23, n.6, p.916-929, 1994.

NATIONAL RESEARCH COUNCIL - NRC. Board on Agriculture. Committee on Animal Nutrition. Subcommittee on Poultry Nutrition. 9.ed. Washington D.C: National Academy Press, 1994. 155p.

NAVARRO, G. Nuevos conseptos de la soya integral en la alimentación avícola. México:Asociación Americana de Soya, 1992. 6p. (Buletim Técnico,102).

NOY, Y.; SKLAN, D. Digestion and absorption in the young chick. Poultry Science, v.74, p.366-373, 1995.

PINHEIRO, L.W. Sojas integral processada pelo calor em rações de frangos de corte. Jaboticabal: Universidade Estadual Paulista, 1993. 75p. Tese (Doutorado em Zootecnia) Universidade Estadual Paulista, 1993.

PUPA, J.M.R.; LEÃO, M. I.; CARVALHO, A. V. et al. Cecectomia em galos sobanestesia local e incisão abdominal. Arquivos Brasileiros deMedicina Veterinária e Zootecnia, v.50, n.5, p.531-535, 1998 .

RODRIGUES, P.B.; ROSTAGNO, H.S.; ALBINO, L.F.T. et al Aminoácidos digestíveis verdadeiros da soja e subprodutos, determinados com galos cecectomizados. Revista Brasileira de Zootecnia, v.31, n.2, p.970-981, 2002.

ROSTAGNO, H.S.; ALBINO, L.F.T.; DONZELE, J.L et al. Tabelas brasileiras para aves e suínos: Composição de alimentos e exigências nutricionais. Viçosa, MG: Universidade Federal de Viçosa, 2000. 141p. 
SAKOMURA, N.K; SILVA, R.; LAURENTZ, A.C et al. Avaliação da soja integral tostada ou extrusada sobre o desempenho de frangos de corte. Revista Brasileira de Zootecnia, v.27, n.3, p.584-594, 1998.

SAKOMURA; N.K; DEL BIANCHI, M.; PIZAURO JR., J.M. et al. Efeito da idade dos frangos de corte na atividade enzimática e digestibilidade dos nutrientes do farelo de soja e da soja integral. Revista Brasileira de Zootecnia,v.33, n.4, p.924-935, 2004.

STATISTICAL ANALYSES SYSTEM - SAS. SAS/STAT: user's guide, version 6.11. ed. Cary: 1996. 842p.

SIBBALD, I.R. A biossay for true metabolizable energy in feedingstuffs. Poultry Science, v.55, n.1, p.303-308, 1976.

SILVA, D.J.; QUEIROZ, A.C. Análise de alimentos: Métodos químicos e biológicos. 3.ed. Viçosa, MG: Universidade Federal de Viçosa, 2002. 165p.

SKLAN, D. Digestion and absorption of lipids in chicks fed triglycerides or free fatty acids: syntesis of monoglycerides in the intestine. Poultry Science, v.58, p.885-889, 1979.

VILÁ, B.; ESTEVE-GARCIA, E. Studies on acid oils and fatty acids for chickens. I. Influence of age, rate inclusion and degree of saturation on fat digestibility and metabolizable energy of acid oils. British Poultry Science, v.37, p.105-117, 1996.

WALDROUP, P.W. Whole soybeans for poultry feeds. World's Poultry Science Journal, v.38, n.1, p.28-35, 1982.
WISEMAN , J.; SALVADOR, F. The influence of free fatty acid content and degree of saturation on the apparent metabolizable energy value of fats fed to broilers. Poultry Science, v.70, p.573-582, 1991.

WOLYNETZ, M.N.; SIBBALD, I.R. Relationships between apparent and true metabolizable energy and the effects of a nitrogen correction. Poultry Science, v.63, n.7, p.13861399, 1984.

YAGHOBFAR, A. Effect of genetic line, sex of birds and the type of bioassay on the metabolisable energy value of maize. British Poultry Science, v.42, p.350-353, 2001.

ZANELLA, I.; SAKOMURA. N.K.; SILVERSIDES, F. G. et al. Effect of enzyme supplementation of broiler diets base on corn and soybeans. Poultry Science, v 78, p. 561-568, 1999.

Recebido em: 19/08/04

Aceito em: 18/04/05 